\title{
Common genetic variation at the IL1RL1 locus regulates IL-33/ST2 signaling
}

\author{
Jennifer E. Ho,, ${ }^{1,2}$ Wei-Yu Chen, ${ }^{3}$ Ming-Huei Chen,,1,4 Martin G. Larson,, ${ }^{1,5}$ Elizabeth L. McCabe, ${ }^{6}$ \\ Susan Cheng, ${ }^{1,3}$ Anahita Ghorbani, ${ }^{7}$ Erin Coglianese, ${ }^{8}$ Valur Emilsson, ${ }^{9}$ Andrew D. Johnson, ${ }^{1}$ \\ Stefan Walter, ${ }^{10,11,12}$ Nora Franceschini, ${ }^{13}$ Christopher J. O'Donnell, 1,7 CARDloGRAM Consortium, \\ CHARGE Inflammation Working Group, Abbas Dehghan, ${ }^{12}$ Chen Lu, ${ }^{5}$ Daniel Levy, ${ }^{1}$ \\ Christopher Newton-Cheh, 1,7,14,15 CHARGE Heart Failure Working Group, Honghuang Lin,1,16 \\ Janine F. Felix, ${ }^{12}$ Eric R. Schreiter, ${ }^{17,18}$ Ramachandran S. Vasan, 1,19 James L. Januzzi, ${ }^{7}$ \\ Richard T. Lee, ${ }^{3}$ and Thomas J. Wang ${ }^{1,7,20}$
}

\begin{abstract}
${ }^{1}$ Framingham Heart Study (FHS) of the National Heart, Lung and Blood Institute and Boston University School of Medicine, Framingham, Massachusetts, USA. ${ }^{2}$ Cardiovascular Medicine Section, Department of Medicine, Boston University School of Medicine, Boston, Massachusetts, USA.

${ }^{3}$ Division of Cardiology, Brigham and Women's Hospital, Harvard Medical School, Boston, Massachusetts, USA. ${ }^{4}$ Department of Neurology, School of Medicine,

${ }^{5}$ Department of Mathematics and Statistics, and ${ }^{6}$ School of Public Health, Boston University, Boston, Massachusetts, USA. ${ }^{7}$ Cardiology Division,

Massachusetts General Hospital, Harvard Medical School, Boston, Massachusetts, USA. ${ }^{\circ}$ Loyola University Health System, Chicago, Illinois, USA

9Icelandic Heart Association, Kopavogur, Iceland. ${ }^{10}$ Department of Society, Human Development, and Health, Harvard School of Public Health,

Boston, Massachusetts, USA. ${ }^{11}$ Department of Public Health and ${ }^{12}$ Department of Epidemiology, Erasmus Medical Center, Rotterdam, The Netherlands. ${ }^{13}$ Department of Epidemiology, University of North Carolina, Chapel Hill, North Carolina, USA. ${ }^{14}$ Broad Institute of Harvard and MIT, Cambridge, Massachusetts, USA. ${ }^{15}$ Cardiovascular Research Center, Massachusetts General Hospital, Boston, Massachusetts, USA. ${ }^{16}$ Computational Biomedicine, Department of Medicine, Boston University School of Medicine, Boston, Massachusetts, USA. ${ }^{17}$ Department of Chemistry, University of Puerto Rico, Rio Piedras, San Juan, Puerto Rico, USA. ${ }^{18} \mathrm{Janelia}$ Farm Research Campus, Howard Hughes Medical Institute,

Ashburn, Virginia, USA. ${ }^{9} \mathrm{C}$ Cardiology and Preventive Medicine, Department of Medicine, Boston University School of Medicine, Boston, Massachusetts, USA. ${ }^{20}$ Division of Cardiovascular Medicine, Department of Medicine, Vanderbilt University, Nashville, Tennessee, USA.
\end{abstract}

\begin{abstract}
The suppression of tumorigenicity 2/IL-33 (ST2/IL-33) pathway has been implicated in several immune and inflammatory diseases. ST2 is produced as 2 isoforms. The membrane-bound isoform (ST2L) induces an immune response when bound to its ligand, IL-33. The other isoform is a soluble protein (sST2) that is thought to be a decoy receptor for IL-33 signaling. Elevated sST2 levels in serum are associated with an increased risk for cardiovascular disease. We investigated the determinants of sST2 plasma concentrations in 2,991 Framingham Offspring Cohort participants. While clinical and environmental factors explained some variation in sST2 levels, much of the variation in SST2 production was driven by genetic factors. In a genome-wide association study (GWAS), multiple SNPs within IL1RL1 (the gene encoding ST2) demonstrated associations with sST2 concentrations. Five missense variants of IL1RL1 correlated with higher sST2 levels in the GWAS and mapped to the intracellular domain of ST2, which is absent in SST2. In a cell culture model, IL1RL1 missense variants increased sST2 expression by inducing IL-33 expression and enhancing IL-33 responsiveness (via ST2L). Our data suggest that genetic variation in $I L 1 R L 1$ can result in increased levels of sST2 and alter immune and inflammatory signaling through the ST2/IL-33 pathway.
\end{abstract}

\section{Introduction}

Suppression of tumorigenicity 2 (ST2) is a member of the IL-1 receptor (IL-1R) family that plays a major role in immune and inflammatory responses. Alternative promoter activation and splicing produces both a membrane-bound protein (ST2L) and a soluble form (sST2) (1). The transmembrane form of ST2 is selectively expressed on Th2- but not Th1-type T cells, and binding of its ligand, IL-33, induces Th2 immune responses $(2,3)$.

Authorship note: Jennifer E. Ho and Wei-Yu Chen contributed equally to this work. Richard T. Lee and Thomas J. Wang contributed equally to this work and are cosenior authors.

Conflict of interest: Measurement of the sST2 assay was performed by Critical Diagnostics Inc. James L. Januzzi has received research grant funding from and has served on the advisory board for Critical Diagnostics Inc. Brigham and Women's Hospital holds patents on ST2, listing Richard T. Lee as inventor.

Note regarding evaluation of this manuscript: Manuscripts authored by scientists associated with Duke University, The University of North Carolina at Chapel Hill, Duke-NUS, and the Sanford-Burnham Medical Research Institute are handled not by members of the editorial board but rather by the science editors, who consult with selected external editors and reviewers.

Citation for this article: J Clin Invest. 2013;123(10):4208-4218. doi:10.1172/JCI67119.
In contrast, the soluble form of ST2 acts as a decoy receptor by sequestering IL-33 (4). The IL-33/ST2 pathway has important immunomodulatory effects $(2,3)$.

Clinically, the ST2/IL-33 signaling pathway participates in the pathophysiology of a number of inflammatory and immune diseases related to Th2 activation, including asthma (5), ulcerative colitis (6), and inflammatory arthritis (7). ST2 expression is also upregulated in cardiomyocytes in response to stress (8) and appears to have cardioprotective effects in experimental studies $(9,10)$. As a biomarker, circulating sST2 concentrations have been linked to worse prognosis in patients with heart failure (11-15), acute dyspnea $(16)$, and acute coronary syndrome $(17,18)$ and also predict mortality and incident cardiovascular events in individuals without existing cardiovascular disease (19).

Both sST2 and its transmembrane form are encoded by IL-1Rlike 1 (IL1RL1). Genetic variation in this pathway has been linked to a number of immune and inflammatory diseases (20). The contribution of IL1RL1 locus variants to interindividual variation in sST2 has not been investigated. The emergence of sST2 as an important predictor of cardiovascular risk and the important role 


\section{Table 1}

Genome-wide significant SNPs (with $\left.r^{2}<0.2\right)$ associated with sST2

\begin{tabular}{|c|c|c|c|c|c|c|c|c|c|}
\hline Chr & SNP & $\begin{array}{l}\text { Position } \\
\text { (NCBI 36.3) }\end{array}$ & $\begin{array}{l}\text { Location } \\
\text { relative to gene }\end{array}$ & $\begin{array}{l}\text { Nearest } \\
\text { gene(s) }\end{array}$ & $\begin{array}{c}\text { Allele } \\
\text { (major/minor) }\end{array}$ & MAF & $\beta^{A}$ & s.e. & $P$ \\
\hline 2 & rs950880 & 102298994 & Intron & IL1RL1 & $\mathrm{C} / \mathrm{A}$ & 0.39 & -0.18 & 0.01 & 7.11E-94 \\
\hline 2 & rs13029918 & 102323723 & Intron, splice site & ILIRL1 & $A / G$ & 0.05 & -0.29 & 0.02 & $1.54 \mathrm{E}-39$ \\
\hline 2 & rs1420103 & 102315064 & Intron & ILIRL1 & $\mathrm{C} / \mathrm{A}$ & 0.23 & 0.13 & 0.01 & $1.01 \mathrm{E}-35$ \\
\hline 2 & rs1014286 & 102515532 & nSNP & SLC9A4 & $A / G$ & 0.36 & 0.11 & 0.01 & $2.61 \mathrm{E}-33$ \\
\hline 2 & rs17639215 & 102319876 & Intron & $I L 1 R L 1$ & $\mathrm{G} / \mathrm{A}$ & 0.13 & 0.15 & 0.01 & $1.71 \mathrm{E}-30$ \\
\hline 2 & rs12999542 & 102331824 & Intron & IL1RL1 & $\mathrm{A} / \mathrm{C}$ & 0.11 & -0.15 & 0.01 & $2.08 \mathrm{E}-27$ \\
\hline 2 & rs13019803 & 102142634 & Intron & $I L 1 R 1$ & $\mathrm{C} / \mathrm{T}$ & 0.11 & -0.14 & 0.02 & $5.95 \mathrm{E}-20$ \\
\hline 2 & rs1558648 & 102176600 & Intron & IL1RL2 & $T / G$ & 0.12 & -0.13 & 0.02 & $3.94 \mathrm{E}-16$ \\
\hline 2 & rs2302612 & 102218140 & nSNP & IL1RL2 & $\mathrm{T} / \mathrm{C}$ & 0.17 & -0.08 & 0.01 & $2.08 \mathrm{E}-11$ \\
\hline 2 & rs11693697 & 102282094 & Intergenic & IL1RL1 & $\mathrm{T} / \mathrm{C}$ & 0.22 & -0.08 & 0.01 & $6.81 \mathrm{E}-11$ \\
\hline 2 & rs11465699 & 102421199 & Intron & IL18RAP & $\mathrm{G} / \mathrm{A}$ & 0.06 & 0.14 & 0.02 & $1.64 \mathrm{E}-08$ \\
\hline
\end{tabular}

A $\beta$ estimate represents estimated change in log-sST2 per minor allele. Chr, chromosome; MAF, minor allele frequency; $\beta$, $\beta$ estimate; SLC9A4, solute carrier family 9 member 4; IL1R1, IL-1R, type I; IL1RL2, IL-1R-like 2.

of the ST2/IL-33 pathway in inflammatory diseases highlight the value of understanding genetic determinants of sST2. The family-based FHS cohort provides a unique opportunity to examine the heritability of sST2 and to identify specific variants involved using a genome-wide association study (GWAS). Thus, we performed a population-based study to examine genetic determinants of sST2 concentrations, coupled with experimental studies to elucidate the underlying molecular mechanisms.

\section{Results}

Clinical characteristics of the 2,991 FHS participants are presented in Supplemental Table 1 (supplemental material available online with this article; doi:10.1172/JCI67119DS1). The mean age of participants was 59 years, and $56 \%$ of participants were women. Soluble ST2 concentrations were higher in men compared with those in women $(P<0.001)$. Soluble ST2 concentrations were

\section{Table 2}

Expression-associated SNPS

$\begin{array}{lcccc}\text { eSNP } & \text { sST2 } \boldsymbol{P} & \text { Tissue } & \text { eSNP } \boldsymbol{P} & \text { Transcript } \\ \text { rs10515921 } & 1.85 \mathrm{E}-25 & \text { Blood } & 2.40 \mathrm{E}-33 & \text { IL18RAP } \\ & & & 9.10 \mathrm{E}-06 & \text { IL18R1 } \\ \text { rs13001325 } & 8.64 \mathrm{E}-94 & \text { Cerebellum } & 1.46 \mathrm{E}-09 & \text { IL18R1 } \\ & & & 1.54 \mathrm{E}-05 & \text { IL1RL1 } \\ \text { rs13001325 } & 8.64 \mathrm{E}-94 & \text { Prefrontal cortex } & 1.95 \mathrm{E}-12 & \text { IL1RL1 } \\ & & & 2.99 \mathrm{E}-12 & \text { IL18R1 } \\ \text { rs13001325 } & 8.64 \mathrm{E}-94 & \text { Visual cortex } & 4.77 \mathrm{E}-10 & \text { IL18R1 } \\ & & & 1.85 \mathrm{E}-07 & \text { IL1RL1 } \\ \text { rs1420101 } & 4.33 \mathrm{E}-92 & \text { Adipose } & 2.53 \mathrm{E}-05 & \text { IL18R1 } \\ \text { rs1420101 } & 4.33 \mathrm{E}-92 & \text { Blood } & 2.08 \mathrm{E}-20 & \text { IL18RAP } \\ \text { rs1420101 } & 4.33 \mathrm{E}-92 & \text { Lymphocytes } & 5.05 \mathrm{E}-06 & \text { IL18R1 } \\ \text { rs1420101 } & 4.33 \mathrm{E}-92 & \text { CD4+ lymphocytes } & 5.01 \mathrm{E}-04 & \text { IL18R1 } \\ \text { rs1420101 } & 4.33 \mathrm{E}-92 & \text { Blood } & 5.50 \mathrm{E}-04 & \text { IL18R1 } \\ \text { rs1946131 } & 2.21 \mathrm{E}-27 & \text { Blood } & 8.50 \mathrm{E}-41 & \text { IL18RAP } \\ & & & 9.40 \mathrm{E}-11 & \text { IL18R1 } \\ \text { rs741285 } & 2.61 \mathrm{E}-33 & \text { Blood } & 2.60 \mathrm{E}-20 & \text { IL18RAP } \\ & & & 7.00 \mathrm{E}-09 & \text { IL18R1 }\end{array}$

Pairwise linkage disequilibrium with top 10 sST2 loci: rs10515921 and rs17639215 $\left(r^{2}=0.94\right)$; rs13001325 and rs950880; rs1420101 and rs950880; rs1946131 and rs12999542; rs741285 and rs1014286 ( $r^{2}=1.0$ for all). positively associated with age, systolic blood pressure, body-mass index, antihypertensive medication use, and diabetes mellitus $(P<0.05$ for all). Together, these variables accounted for $14 \%$ of the variation in sST2 concentrations. The duration of hypertension or diabetes did not materially influence variation in sST2 concentrations. After additionally accounting for inflammatory conditions, clinical variables accounted for $14.8 \%$ of sST2 variation.

Heritability of SST2. The age- and sex-adjusted heritability $\left(\mathrm{h}^{2}\right)$ of sST2 was $0.45\left(P=5.3 \times 10^{-16}\right)$, suggesting that up to $45 \%$ of the variation in sST2 not explained by clinical variables was attributable to genetic factors. Multivariable adjustment for clinical variables previously shown to be associated with SST2 concentrations (21) did not attenuate the heritability estimate (adjusted $h^{2}=0.45, P=8.2$ $\left.\times 10^{-16}\right)$. To investigate the influence of shared environmental factors, we examined the correlation of sST2 concentrations among 603 spousal pairs and found no significant correlation $(r=0.05, P=0.25)$. Genetic correlates of sST2. We conducted a GWAS of circulating sST2 concentrations. Quantile-quantile, Manhattan, and regional linkage disequilibrium plots are shown in Supplemental Figures 1-3. There were 388 SNPs associated with sST2 concentrations at $P<5 \times 10^{-8}$, all on chromosome 2q12.1 (Supplemental Table 2). All genome-wide significant SNPs were located in a 400-kb linkage disequilibrium block that included IL1RL1 (the gene encoding ST2), IL1R1, IL1RL2, IL18R1, IL18RAP, and SLC9A4 (Figure 1). Results for 11 genome-wide significant "independent" SNPs, defined as pairwise $r^{2}<0.2$, are shown in Table 1. In aggregate, these 11 "independent" genome-wide significant SNPs across the IL1RL1 locus accounted for $36 \%$ of heritability of sST2. In conditional analyses, 4 out of the 11 SNPs remained genome-wide significant, independent of each other (rs950880, rs13029918, rs1420103, and rs17639215), all within the IL1RL1 locus. The most significant SNP (rs950880, $P=7.1 \times 10^{-94}$ ) accounted for $12 \%$ of the residual interindividual variability in circulating sST2 concentrations. Estimated mean sST2 concentrations were $43 \%$ higher in major homozygotes (CC) compared with minor homozygotes (AA). Three loci 


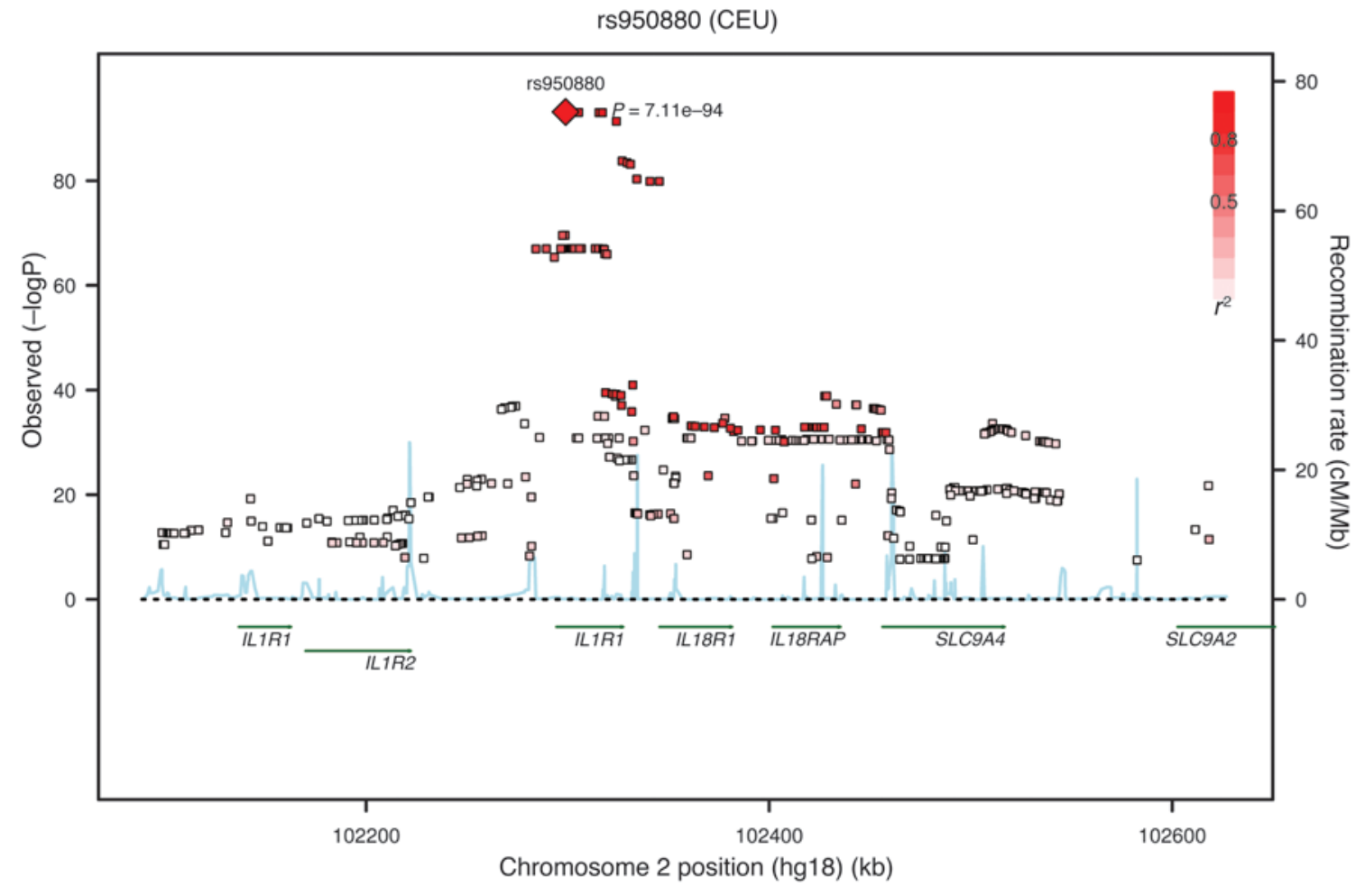

Figure 1

Regional association plot of SST2 SNPs with $P<5 \times 10^{-8}$. "CEU" refers to Utah residents with northern and western European ancestry from the $\mathrm{CEPH}$ collection. Recombination rates are superimposed in blue, and gene regions are in green.

outside of the IL1RL1 locus had suggestive associations with SST2 $\left(P<1 \times 10^{-6}\right)$ and are displayed in Supplemental Table 3 .

In silico association with expression SNPs. The top $10 \mathrm{sST} 2 \mathrm{SNPS}$ (among 11 listed in Table 1) were explored in collected gene expression databases. There were 5 genome-wide significant sST2 SNPs associated with gene expression across a variety of tissue types (Table 2). Specifically, rs 13001325 was associated with IL1RL1 gene expression (the gene encoding both soluble and transmembrane ST2) in several subtypes of brain tissue (prefrontal cortex, $P=1.95 \times 10^{-12}$; cerebellum, $P=1.54 \times 10^{-5}$; visual cortex, $\left.P=1.85 \times 10^{-7}\right)$. The CC genotype of rs 13001325 was associated with a higher IL1RL1 gene expression level as well as a higher circulating sST2 concentration when compared with the TT genotype (Supplemental Figure 4). Other ST2 variants were significantly associated with IL18RAP $\left(P=8.50 \times 10^{-41}\right.$, blood $)$ and IL18R1 gene expression $\left(P=2.99 \times 10^{-12}\right.$, prefrontal cortex $)$.

In silico association with clinical phenotypes in published data. The $\mathrm{G}$ allele of rs1558648 was associated with lower sST2 concentrations in the FHS $\left(0.88\right.$-fold change per G allele, $\left.P=3.94 \times 10^{-16}\right)$ and higher all-cause mortality (hazard ratio [HR] 1.10 per G allele, 95\% CI 1.03-1.16, $P=0.003$ ) in the CHARGE consortium, which observed 8,444 deaths in 25,007 participants during an average follow-up of 10.6 years (22). The T allele of rs 13019803 was associated with lower sST2 concentrations in the FHS (0.87-fold change per G allele, $P=5.95 \times 10^{-20}$ ), higher mortality in the CHARGE consortium (HR 1.06 per C allele, 95\% CI 1.01-1.12, $P=0.03$ ), and higher risk of coronary artery disease (odds ratio 1.06, 95\% CI 1.00-1.11, $P=0.035)$ in the CARDIoGRAM consortium, which included
22,233 individuals with coronary artery disease and 64,762 controls (23). In relating sST2 SNPs to other clinical phenotypes (including blood pressure, body-mass index, lipids, fasting glucose, natriuretic peptides, C-reactive protein, and echocardiographic traits) in previously published studies, we found nominal associations with C-reactive protein for 2 SNPs (Supplemental Table 4).

Putative functional variants. Using GeneCruiser, we examined nonsynonymous SNPs (nSNPs) (missense variants) that had at least suggestive association with sST2 $\left(P<1 \times 10^{-4}\right)$, including SNPs that served as proxies $\left(r^{2}=1.0\right)$ for nSNPs within the 1000 Genomes Pilot 1 data set (ref. 24 and Table 3). There were 6 missense variants located within the IL1RL1 gene, 5 of which had genome-wide significant associations with sST2 concentrations, including rs6749114 (proxy for rs10192036, Q501K), rs4988956 (A433T), rs10204137 (Q501R), rs10192157 (T549I), rs10206753 (L551S), and rs1041973 (A78AE). Base substitutions and corresponding amino acid changes for these coding mutations are listed in Table 3. In combination, these 6 missense mutations accounted for $5 \%$ of estimated heritability, with an effect estimate of 0.23 (standard error [s.e.] 0.02, $P=2.4 \times 10^{-20}$ ). When comparing major homozygotes with minor homozygotes, the estimated sST2 concentrations for these missense variants differed by $11 \%$ to $15 \%$ according to genotype (Supplemental Table 5). In conditional analyses, intracellular and extracellular variants appeared to be independently associated with sST2. For instance, in a model containing rs4988956 (A433T) and rs1041973 (A78E), both SNPs remained significantly associated with sST2 $\left(P=2.61 \times 10^{-24}\right.$ and $P=7.67 \times 10^{-15}$, respectively). In total, missense vari- 
A

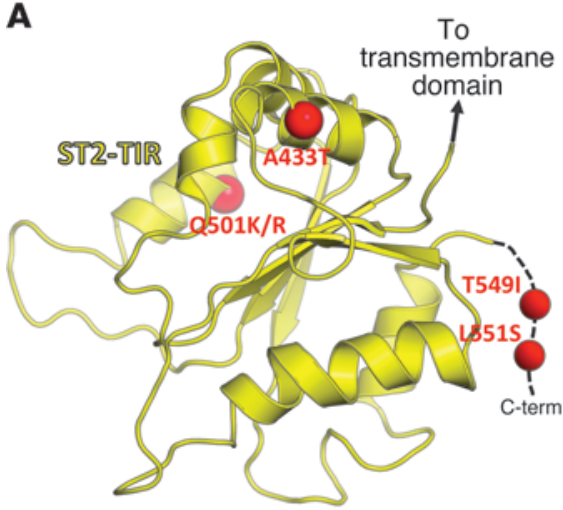

B

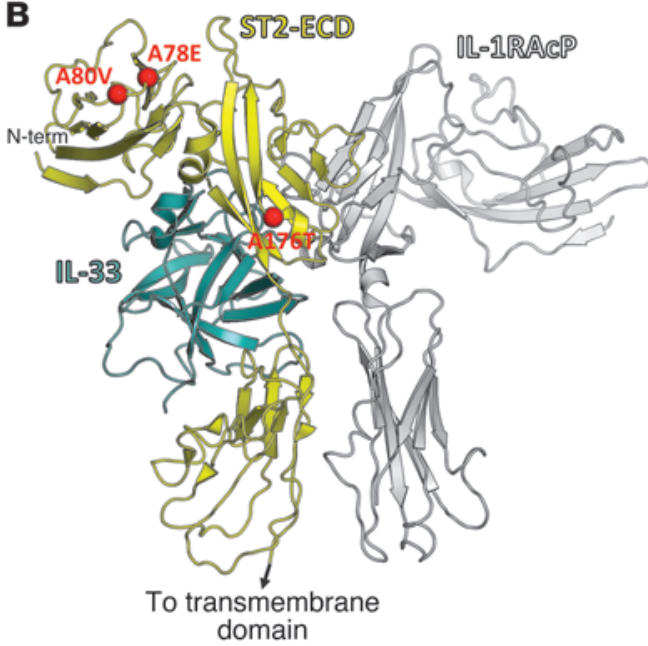

\section{Figure 2}

Models of ST2 illustrate IL1RL1 missense variant locations. Models of the (A) intracellular TIR domain (ST2-TIR) and the (B) extracellular domain (ST2-ECD) of ST2 (protein data bank codes 3040 and 1T3G, respectively). Domains of ST2 are shown in yellow, with identified missense SNP positions represented as red spheres and labels. Note that positions 549 and 551 are near the C terminus of ST2, which is not defined in the crystal structure (protein data bank ID 1T3G, shown as dashed black line in A). Arrows point toward the transmembrane domain, which is also not observed in crystal structures. ants added little to the proportion of sST 2 variance explained by the 11 genome-wide significant nonmissense variants listed in Table 1. In relating these 6 missense variants to other clinical phenotypes in large consortia, we found an association with asthma for 4 out of the 6 variants (lowest $P=4.8 \times 10^{-12}$ for rs10204137) (25).

Homology map of IL1RL1 missense variants and ST2 structure. Of the 6 missense variants mapping to $I L 1 R L 1,5$ were within the cytoplasmic Toll/IL-1R (TIR) domain of the transmembrane ST2 receptor (Figure 2A), and these intracellular variants are thus not part of the circulating sST2 protein. Of these cytoplasmic domain variants, A433T was located within the "box 2" region of sequence conservation, described in the IL-1R1 TIR domain as important for IL-1 signaling (26). Q501R/K was within a conserved motif called "box 3," but mutants of IL-1R1 in box 3 did not significantly affect IL-1 signaling in previous experiments (26). Both T549I and L551S were near the $\mathrm{C}$ terminus of the transmembrane ST2 receptor and were not predicted to alter signaling function based on previous experiments with the IL-1R (26). The A78E SNP was located within the extracellular domain of ST2 and is thus present in both the SST2 isoform and the transmembrane ST2 receptor. In models of the ST2/IL-33/IL-1RAcP complex derived from a crystal structure of the IL-1RII/IL-1 $\beta /$ IL-1RAcP complex (protein data bank ID 1T3G and 3O4O), A78E was predicted to be located on a surface loop within the first immunoglobulin-like domain (Figure 2B), distant from the putative IL-33 binding site or the site of interaction with IL-1RAcP. There were 2 rare extracellular variants that were not captured in our GWAS due to low minor allele frequencies (A80E, MAF 0.008; A176T, MAF 0.002). Both were distant from the IL-33 binding site on homology mapping and unlikely to affect IL-33 binding.

Functional effects of IL1RL1 missense variants on sST2 expression and promoter activity. Since 5 of the IL1RL1 missense variants associated with SST2 levels mapped to the intracellular domain of ST2L and hence are not present on sST2 itself, we hypothesized that these missense variants exert effects via intracellular mechanisms downstream of ST2 transmembrane receptor signaling to regulate sST2 levels. To investigate the effect of IL1RL1 missense variants (identified by GWAS) on sST2 expression, stable cell lines expressing WT ST2L, IL1RL1 variants (A78E, A433T, T549I,

Table 3

Putative functional variants with at least suggestive association with SST2

\begin{tabular}{|c|c|c|c|c|c|c|c|c|c|}
\hline Chr & $\begin{array}{c}\text { nSNP } \\
\text { (or proxy) }\end{array}$ & Position & $\begin{array}{c}\text { Gene } \\
\text { (NCBI 36.3) }\end{array}$ & $\begin{array}{c}\text { Allele } \\
\text { (major/minor) }\end{array}$ & $\begin{array}{l}\text { Amino acid } \\
\text { change }\end{array}$ & MAF & $\beta^{A}$ & s.e. & $P$ \\
\hline 2 & rs6749114B & 102334019 & IL1RL1 & $\mathrm{A} / \mathrm{C}$ & Q501K & 0.38 & 0.08 & 0.01 & $3.54 \mathrm{E}-17$ \\
\hline 2 & rs4988956 & 102334439 & IL1RL1 & $\mathrm{G} / \mathrm{A}$ & A433T & 0.38 & 0.08 & 0.01 & $3.66 \mathrm{E}-17$ \\
\hline 2 & rs10204137 & 102334644 & ILIRLI & $A / G$ & Q501R & 0.38 & 0.08 & 0.01 & $3.66 \mathrm{E}-17$ \\
\hline 2 & rs10192157 & 102334788 & ILIRLI & $\mathrm{C} / \mathrm{T}$ & T549I & 0.38 & 0.08 & 0.01 & $4.06 \mathrm{E}-17$ \\
\hline 2 & rs10206753 & 102334794 & ILIRLI & $\mathrm{T} / \mathrm{C}$ & L551S & 0.38 & 0.08 & 0.01 & 4.33E-17 \\
\hline 2 & rs1041973 & 102321900 & ILIRLI & $\mathrm{C} / \mathrm{A}$ & A78E & 0.26 & -0.05 & 0.01 & $2.15 \mathrm{E}-07$ \\
\hline 2 & rs1014286 & 102515532 & SLC9A4 & $A / G$ & G784S & 0.36 & 0.11 & 0.01 & $2.61 \mathrm{E}-33$ \\
\hline 2 & rs2241132 & 102170467 & IL1RL2 & $\mathrm{C} / \mathrm{A}$ & $\mathrm{A} 124 \mathrm{G}$ & 0.10 & -0.13 & 0.02 & $2.68 \mathrm{E}-15$ \\
\hline 2 & rs2302612 & 102218140 & IL1RL2 & $\mathrm{T} / \mathrm{C}$ & L550P & 0.17 & -0.08 & 0.01 & $2.08 \mathrm{E}-11$ \\
\hline 6 & rs3799854B & 46907968 & MEP1A & $\mathrm{T} / \mathrm{C}$ & T726M & 0.38 & -0.04 & 0.01 & 4.18E-05 \\
\hline 6 & rs2274658 & 46909030 & MEP1A & $\mathrm{G} / \mathrm{T}$ & V469L & 0.40 & -0.04 & 0.01 & $5.82 \mathrm{E}-05$ \\
\hline 1 & rs1009668 & 224107027 & TMEM63A & $\mathrm{C} / \mathrm{T}$ & V622M & 0.09 & -0.06 & 0.02 & $8.22 \mathrm{E}-05$ \\
\hline
\end{tabular}


$\mathbf{A}$

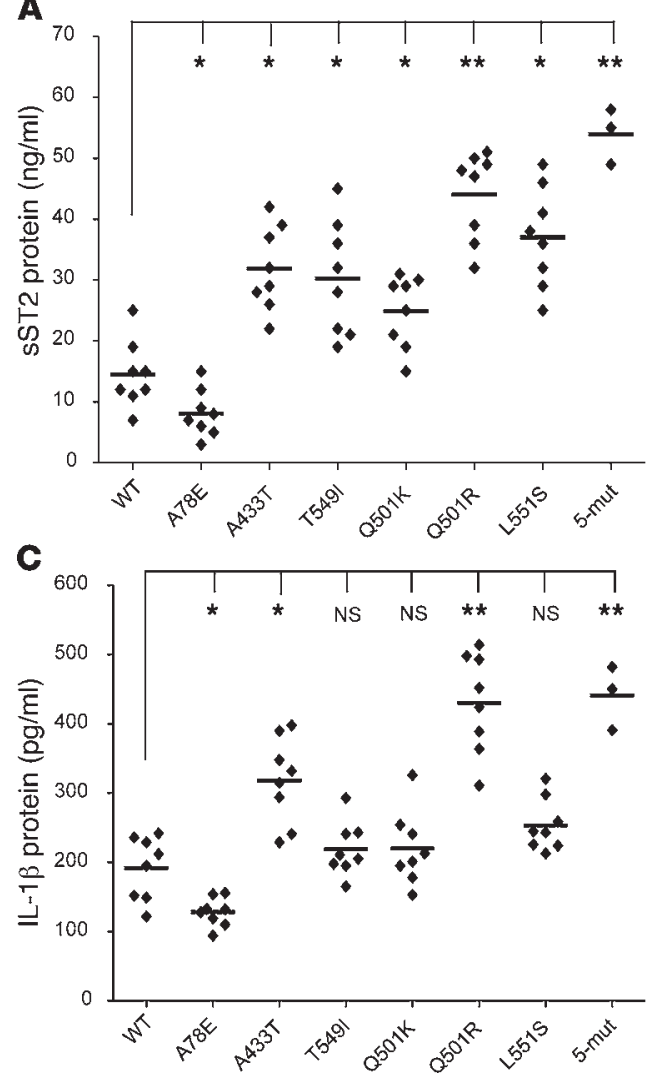

B

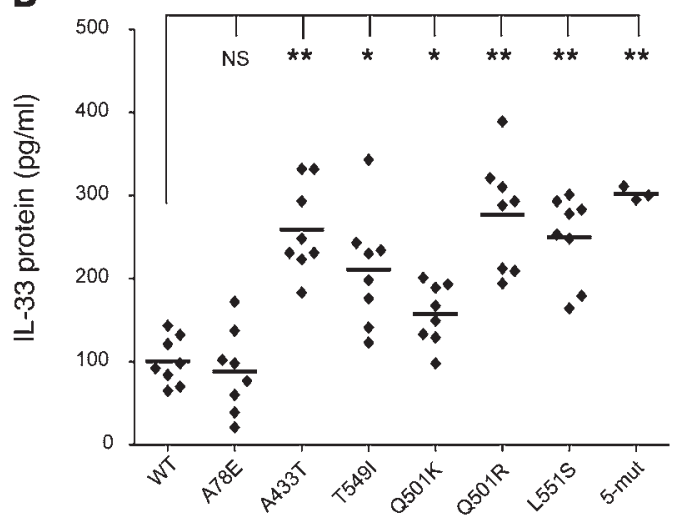

D

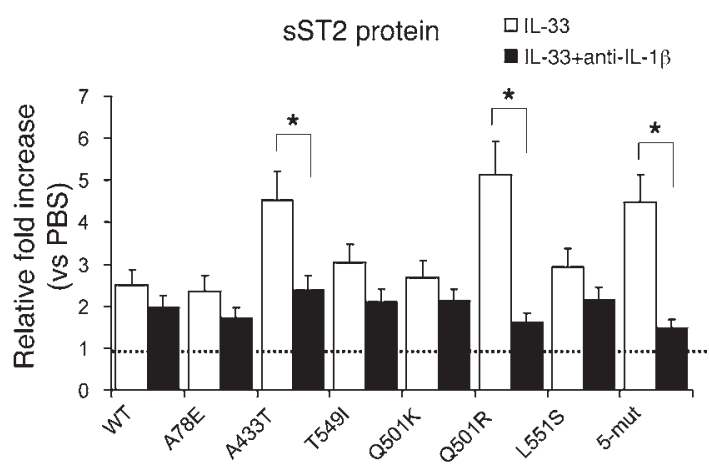

Figure 3

IL1RL1 intracellular missense variants resulted in higher SST2 and IL-33. Media from KU812 cells expressing WT and IL1RL1 missense variants were collected for ELISA analysis of (A) SST2, (B) IL-33, and (C) IL-1 $\beta$ levels. Horizontal bars indicate mean values, and symbols represent individual variants. ${ }^{\star} P<0.05,{ }^{\star \star} P<0.01$ vs. WT. (D) Effect of anti-IL-1 $\beta$ mAb on IL-33-induced SST2 expression. Dashed line indicates PBS-treated cells as referent group. Error bars represent mean \pm SEM from 2 independent experiments. ${ }^{*} P<0.05$ vs. IL-33.

Q501K, Q501R, and L551S), and a construct containing the 5 IL1RL1 intracellular domain variants (5-mut) were generated. Expression of ST2L mRNA and protein (detected in membrane fractions) was confirmed (Supplemental Figures 5 and 6). Eight different stable clones in each group were analyzed to reduce bias from clonal selection.

Intracellular domain variants (A433T, T549I, Q501K, Q501R, L551S, and 5-mut), but not the extracellular domain variant (A78E), were associated with increased basal sST2 expression when compared with WT expression ( $P<0.05$ for all, Figure $3 \mathrm{~A}$ ). sST2 expression was highest in the 5-mut construct, suggesting that intracellular ST2L variants cooperatively regulate sST2 levels. This same pattern was consistent across different cell types (U937, Jurkat T, and A549 cells; Supplemental Figure 7). These findings suggest that intracellular domain variants of the transmembrane ST2 receptor may functionally regulate downstream signaling.

IL1RL1 transcription may occur via two alternative promoters (proximal vs. distal), which leads to differential expression of the soluble versus membrane-bound ST2 proteins (1). Similar to the sST2 protein expression results above, the intracellular domain variants, but not the extracellular domain variant, were associated with higher basal proximal promoter activity. Distal promoter activity was also increased for most intracellular domain variants (Supplemental Figure 8).
IL1RL1 intracellular missense variants resulted in higher IL-33 protein levels. In addition to upregulation of sST2 protein levels, IL1RL1 intracellular missense variants caused increased basal IL-33 protein expression (Figure 3B), suggesting a possible autoregulatory loop whereby IL-33 signaling positively induces sST2 expression. IL-33 induced sST2 protein expression in cells expressing both WT and IL1RL1 missense variants. Interestingly, this effect was particularly pronounced in the A433T and Q501R variants (Supplemental Figure 9A).

Enhanced $I L-33$ responsiveness is mediated by $I L-1 \beta$ in $A 433 T$ and Q501R variants. Because IL-1 $\beta$ can induce sST2 (27), we hypothesized that IL-1 $\beta$ may increase IL-33 responsiveness in the A433T and Q501R variants. The addition of exogenous IL-1 $\beta$ induced sST2 protein expression in all IL1RL1 variants with no difference compared with WT cells (Supplemental Figure 9B). However, basal IL-1 $\beta$ levels were only elevated in A433T, Q501R, and 5-mut constructs (Figure 3C). Further, preincubation with anti-IL-1 $\beta \mathrm{mAb}$ eliminated the enhanced IL-33 responsiveness in A433T, Q501R, and 5 -mut variants $(P<0.05$, Figure $3 \mathrm{D})$. Last, induction of sST2 by IL-33 was blocked by anti-ST $2 \mathrm{mAb}(P<0.05$, Supplemental Figure 10). These findings indicate that IL-33-induced sST2 expression in A433T and Q501R is mediated through IL-1 $\beta$ and transmembrane ST2 receptor signaling.

Interaction among $I L-33, s S T 2$, and $I L-1 \beta$. We further determined 
A
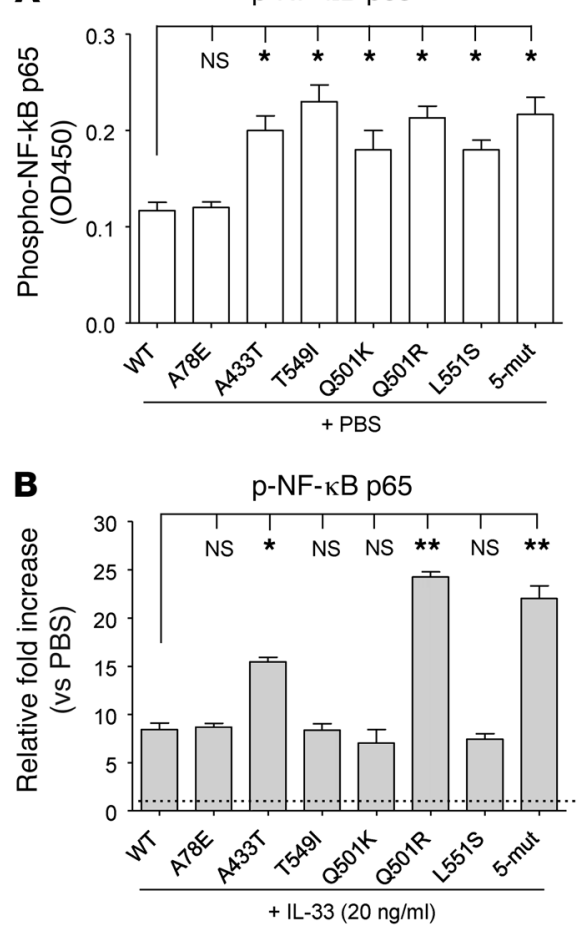

C

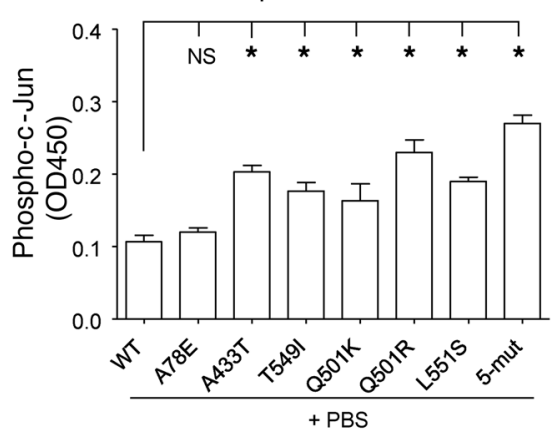

D

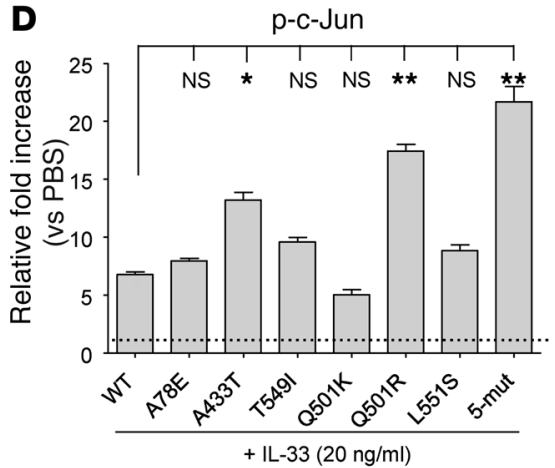

$\mathbf{E}$
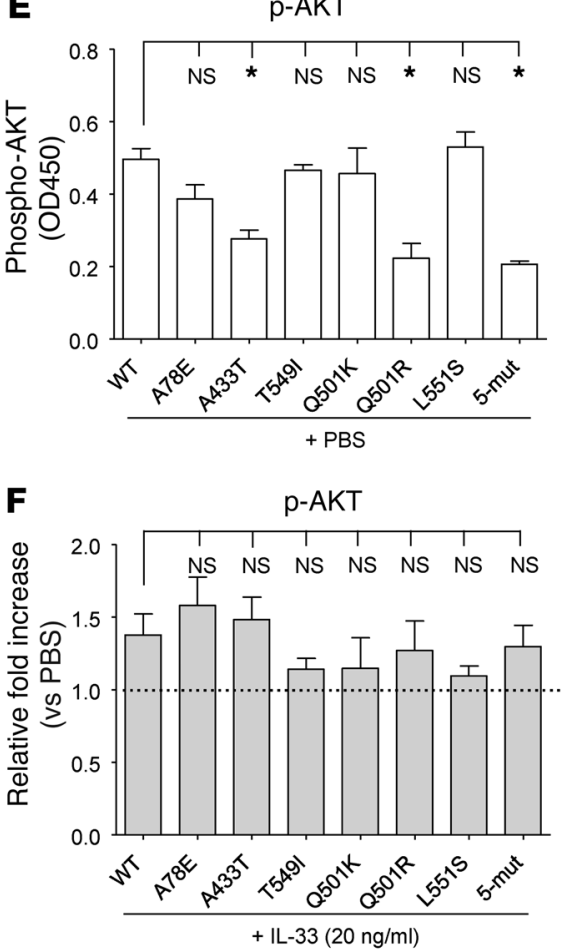

Figure 4

IL1RL1 missense variants modulated ST2 signaling pathways. KU812 cells expressing WT or IL1RL1 variants were treated with PBS or IL-33. Levels of the following phosphorylated proteins were detected in cell lysates using ELISA: (A and B) phospho-NF-KB p65; (C and D) phosphoC-Jun activity; and (E and F) phospho-AKT. (A, C, and E) White bars represent basal levels, and (B, D, and F) gray bars represent relative fold increase (compared with PBS-treated group) after IL-33 treatment. ${ }^{*} P<0.05$ vs. WT; ${ }^{* \star} P<0.01$ vs. PBS-treated group. Dashed line in B, D, and $\mathbf{F}$ represents PBS-treated cells as referent group. Error bars represent mean \pm SEM from 2 independent experiments.

the interaction of IL-33, sST2, and IL-1 $\beta$ in the regulatory loop. Inhibition of IL-1 $\beta$ by anti-IL-1 $\beta$ mAb reduced basal expression of sST2 (Supplemental Figure 11A). Blocking of IL-33 by sST2 did not reduce the induction of IL-1 $\beta$ levels by the IL1RL1 variants (Supplemental Figure 11B). Furthermore, inhibition of IL-1 $\beta$ by anti-IL-1 $\beta$ reduced the basal IL-33 levels. IL-33 itself upregulated sST2 levels, which in turn reduced IL-33 levels (Supplemental Figure 11C). Our results revealed that both IL-33 and IL-1 $\beta$ drive sST2 expression and that IL- $1 \beta$ acts as an upstream inducer of IL-33 and maintains IL-33 expression by intracellular IL1RL1 variants (Supplemental Figure 11D). This suggests that IL1RL1 variants upregulated SST2 mainly through IL-33 autoregulation and that the enhanced IL-33 responsiveness by A433T and Q501R was mediated by IL-1 $\beta$ upregulation.

IL1RL1 missense variants modulate ST2 signaling pathways. The effect of IL1RL1 missense variants on known ST2 downstream regulatory pathways, including NF-KB, AP-1/c-Jun, AKT, and STAT3 (28-32), was examined in the presence and absence of IL-33 (Figure 4 and Supplemental Figure 12). The IL1RL1 intracellular missense variants (A433T, T549I, Q501K, Q501R, and L551S) were associated with higher basal phospho-NF-KB p65 and phospho-c-Jun levels (Figure 4, A and B). Consistent with enhanced IL-33 responsiveness in A433T and Q501R cells, levels of IL-33-induced NF-KB and c-Jun phosphorylation were enhanced in these 2 variants (Figure 4, $\mathrm{B}$ and $\mathrm{D}$ ). In contrast, $\mathrm{A} 433 \mathrm{~T}$ and $\mathrm{Q} 501 \mathrm{R}$ variants showed lower basal phospho-AKT levels (Figure 4E). IL-33 did not directly reg- ulate AKT activation, since phospho-AKT levels were not affected with or without IL-33 stimulation (Figure 4F). IL1RL1 missense variants did not affect STAT3 signaling (Supplemental Figure 12). These results reveal increased activation of basal NF- $\mathrm{KB}$ and AP-1 associated with upregulation of SST2 expression by IL1RL1 intracellular variants. In addition, reduced AKT signaling by A433T and Q501R variants might be associated with enhanced IL-33 responsiveness.

We also found that inhibition of NF-KB and AP-1 reduced IL1RL1 proximal promoter activity, whereas distal promoter activity was inhibited by AP-1 blockade but not NF-кB inhibition. This suggests that both NF-kB and AP-1 are important for proximal promoter activation and that AP-1 is important for distal promoter activation by the intracellular IL1RL1 variants (Supplemental Figure 13).

Mal and MYD88 are required for intracellular IL1RL1 variant-induced sST2 expression. We also analyzed the subcellular localization of ST2L in IL1RL1 variants. ST2L was detected predominantly in the membrane fraction, and SST2 was detected in the cytosol fraction. The intracellular IL1RL1 variants upregulated cytosolic SST2 levels as well as the sST2 levels in the culture media. In addition, A433T and Q501R caused higher membrane ST2L expression (Supplemental Figure 6). To test the possibility that IL1RL1 variants interact with Mal, MYD88, and the PI3K subunit, we performed coimmunoprecipitation assays and found that all of the IL1RL1 variants interacted with Mal and MYD88. In addition, binding of Mal to ST2L was increased by the intracellular variants. Only 
A

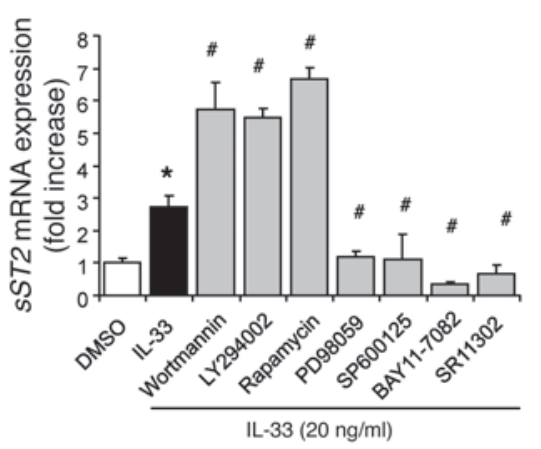

B

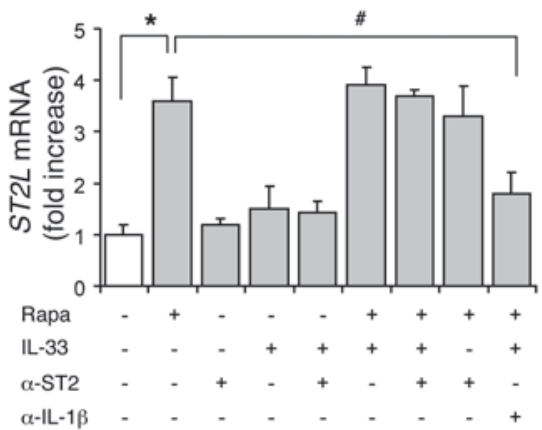

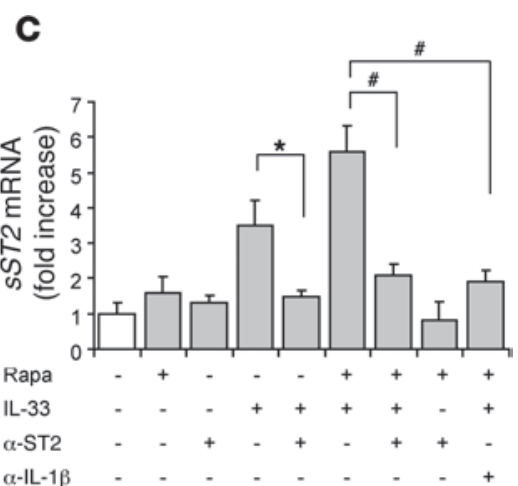

IL-33
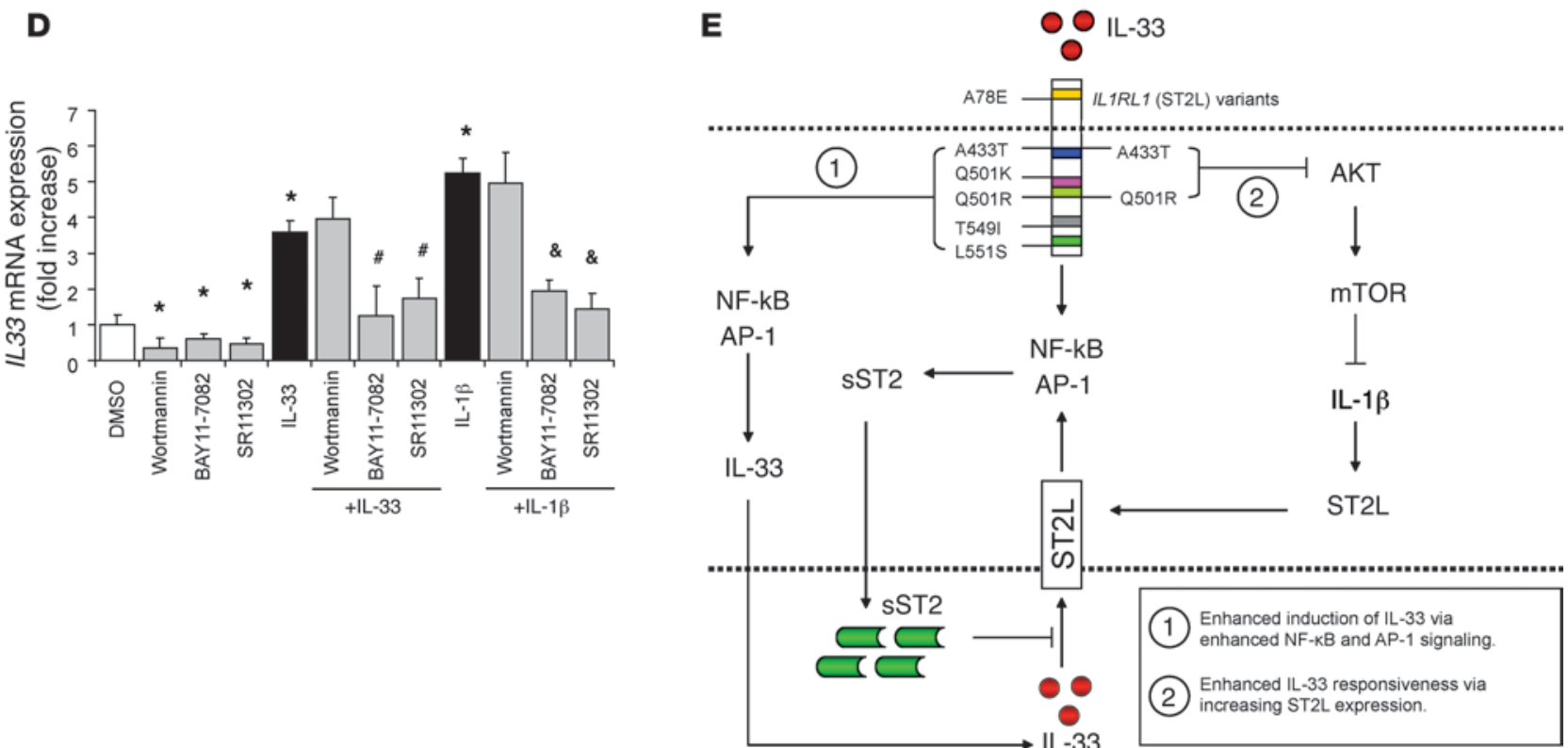

\section{Figure 5}

IL-33-induced sST2 expression is enhanced with mTOR inhibition and occurs via ST2L-dependent signaling. (A) sST2 mRNA expression in KU812 cells after treatment with DMSO, IL-33, or IL-33 plus signal inhibitors (wortmannin, LY294002, rapamycin, PD98059, SP60125, BAY11-7082, or SR11302). (B) ST2L mRNA and (C) SST2 mRNA expression in KU812 cells treated with PBS (white columns), rapamycin (rapa), anti-ST2 mAb, IL-33, IL-33 plus anti-ST2, IL-33 plus rapamycin, IL-33 plus rapamycin plus anti-ST2 mAb, or rapamycin plus anti-ST2. (D) IL33 mRNA expression in KU812 cells after treatment with DMSO, signal inhibitors, IL-33 plus signal inhibitors, and IL-1 $\beta$ plus signal inhibitors. ${ }^{*} P<0.05$ vs. PBS-treated group; $\# P<0.05$ vs. IL-33-treated group; \& $P<0.05$ vs. IL-1 $\beta$-treated group. Error bars represent mean \pm SEM from 2 independent experiments. (E) A schematic model illustrating the regulation of SST2 expression by IL1RL1 missense variants through enhanced induction of IL-33 via enhanced NF-KB and AP-1 signaling and enhanced IL-33 responsiveness via increasing ST2L expression.

A433T and Q501R interacted with the PI3K-p85 subunit, indicating that these $2 I L 1 R L 1$ variants may regulate the PI3K/AKT signaling pathway through the interaction of the ST2L intracellular domain with the PI3K-p85 subunit (Supplemental Figure 14).

We further examined the role of Mal, MYD88, and PI3K-p85 in sST2 induction by IL1RL1 variants. Knockdown of Mal and MYD88 by siRNA completely inhibited sST2 induction by intracellular IL1RL1 variants, indicating that Mal and MYD88 are necessary for ST2L-mediated SST2 induction. Knockdown of PI3K-p85 had no effect on IL-33-induced sST2, whereas knockdown of PI3K-p85 blocked A433T, Q501R, and 5-mut-enhanced IL-33 responsiveness (Supplemental Figure 15). These results suggest that the increased binding of Mal to the ST2L intracellular domain mediates sST2 expression seen in intracellular IL1RL1 variants. Furthermore, the recruitment of PI3K-p85 to A433T and Q501R mediates the enhanced IL-33 responsiveness.

IL-33-induced SST2 expression is reciprocally regulated by different signaling pathways. IL1RL1 intracellular variants caused increased basal activation of NF- $\mathrm{KB}$ and AP-1 and lower basal AKT phosphorylation in A433T and Q501R variants (Figure 4). We hypothesized that SST2 is reciprocally regulated by NF- $\mathrm{B}, \mathrm{AP}-1$, and AKT pathways, and thus we investigated the role of $\mathrm{PI} 3 \mathrm{~K} / \mathrm{AKT} / \mathrm{mTOR}$, ERK/NF- $\mathrm{B}$, and JNK/AP-1 pathways on IL-33-induced sST2 expression. Inhibitors of PI3K and mTOR further enhanced IL-33-induced sST2 expression (Figure 5A). In contrast, inhibitors of ERK, JNK, NF- $\mathrm{KB}$, and AP-1 abrogated IL-33-induced SST2 expression, indicating that NF- $\mathrm{KB}$ and AP-1 are required for IL-33-induced sST2 expression (Figure 5A). These data 
reveal reciprocal regulation of IL-33-induced sST2 expression by $\mathrm{PI} 3 \mathrm{~K} / \mathrm{AKT} / \mathrm{mTOR}, \mathrm{ERK} / \mathrm{NF}-\mathrm{KB}$, and JNK/AP-1 pathways.

Inbibition of the $P I 3 K / A K T / m T O R$ axis increases IL-33 responsiveness and SST2 expression through IL-1ß-mediated ST2L induction. Rapamycin has been shown to trigger ST2L expression through upregulating IL-1 $\beta(30)$. We found that inhibition of mTOR with rapamycin specifically induced ST2L but not SST2 expression in the absence of IL-33 (Figure 5, B and C). This effect was blocked by anti-IL-1 $\beta$ $\mathrm{mAb}$, suggesting that IL- $1 \beta$ mediated rapamycin-triggered ST2L expression (Figure 5B). In addition, rapamycin enhanced IL-33induced sST2 expression, an effect that was blocked by anti-IL-1 $\beta$ and anti-ST2 (Figure 5C). These results suggest that inhibition of the $\mathrm{PI} 3 \mathrm{~K} / \mathrm{AKT} / \mathrm{mTOR}$ axis can increase IL-33 responsiveness via IL-1 $\beta$ triggered endogenous ST2L expression. This may be the underlying mechanism of enhanced IL-33 responsiveness in the A433T and Q501R variants, in which we found reduced AKT signaling.

$N F-\kappa B$ and $A P-1$ are required for $I L-33$ expression. To determine the mechanism for increased IL-33 expression in the presence of IL1RL1 intracellular variants, we examined the role of PI3K/AKT, $\mathrm{NF}-\kappa \mathrm{B}$, and AP-1 in the regulation of IL-33 expression. Inhibition of PI3K, NF- $\mathrm{KB}$, and AP-1 reduced basal IL-33 expression $(P<0.05$; Figure 5D). IL33 mRNA expression was induced by IL-1 $\beta$ and IL-33 itself $(P<0.05)$, which in turn was blocked by inhibition of NF- $\mathrm{B}$ and AP-1 but not affected by inhibition of PI3K/AKT. This indicated that NF- $\mathrm{KB}, \mathrm{AP}-1$, and $\mathrm{PI} 3 \mathrm{~K} / \mathrm{AKT}$ signals are required for basal IL-33 expression, whereas only NF- $\mathrm{KB}$ and AP-1 are necessary for IL-33 induction after IL-1 $\beta$ and IL-33 stimulation.

In summary, our experiments demonstrate that IL-33 induces sST2 expression through NF- $\mathrm{BB}$ and AP-1 and that the IL1RL1 intracellular domain variants regulate IL-33-induced sST2 expression via 2 mechanisms: (a) enhanced induction of IL-33 via enhanced NF- $\mathrm{KB}$ and AP-1 signaling and (b) enhanced IL-33 responsiveness via increasing ST2L expression (Figure 5E).

\section{Discussion}

Our findings indicate that genetic factors account for up to $40 \%$ of the interindividual variability in circulating sST2 concentrations. This substantially exceeds the amount of variation in SST2 attributable to measured clinical and environmental factors $(\sim 14 \%)$, although this may be underestimated due to unmeasured clinical factors. Common genetic variants within or near the IL1RL1 gene alone accounted for more than $10 \%$ of the phenotypic variation in sST2, nearly as much as all measured clinical variables together. We mapped intracellular IL1RL1 missense variants associated with circulating sST2 concentrations in our GWAS to the TIR domain, a region important in downstream signaling. In molecular function studies, we demonstrate that these IL1RL1 missense variants expressed in membrane-bound ST2 (ST2L) but not expressed in its soluble form (sST2) can induce sST2 expression and that this occurs via a direct effect on ST2L expression and downstream signaling. Thus, genetic variants that change intracellular ST2 transmembrane signaling can induce human sST2, revealing a new pathway in immune and inflammatory regulation.

The ST2/IL-33 pathway plays a major role in immune and inflammatory responses by inducing $\mathrm{Th} 2 \mathrm{immune}$ responses $(2,3)$. Clinically, ST2 appears to contribute to the pathophysiology of a number of inflammatory and immune diseases related to Th2 activation, including asthma (5), ulcerative colitis (6), and inflammatory arthritis (7). Additionally, ST2 is emerging as an important mediator of cardiac remodeling as well as a valuable prog- nostic marker in cardiovascular disease (11-13, 15, 17, 33, 34). Understanding the genetic determinants of sST2 may lend important insights into regulation of the SST2 pathway and may lead to the discovery of novel therapeutic targets for the treatment of immune and cardiovascular disease.

The majority of sST2 gene variants in our study were located within or near ILIRL1, the gene coding for both transmembrane ST2 and SST2. IL1RL1 resides within a linkage disequilibrium block of $400 \mathrm{~kb}$ on chromosome $2 \mathrm{q} 12$, a region that includes a number of other cytokines, including IL-18 receptor 1 (IL18R1) and IL-18 receptor accessory protein (IL18RAP). Polymorphisms in this gene cluster have been associated previously with a number of immune and inflammatory conditions, including asthma (35), celiac disease (36), and type 1 diabetes mellitus (37). Many of these variants were associated with sST2 concentrations in our analysis (Supplemental Table 6). The immune effects of ST2 are corroborated by experimental evidence: membrane-bound ST2 is selectively expressed on Th2- but not Th1-type T helper cells, and activation of the ST2/IL-33 axis elaborates Th2 responses $(2,3)$. In general, the allergic phenotypes above are thought to be Th2mediated processes, in contrast to atherosclerosis, which appears to be a Th1-driven process (38).

How these genetic variants differentially relate to these previously described immune and inflammatory diseases compared with cardiovascular disease is unknown. We found 2 variants with suggestive associations with clinical phenotypes. The directionality suggests that variants with lower sST 2 concentrations may be associated with higher C-reactive protein and adverse outcomes, but these data should be considered hypothesis generating. This association is counter to clinical observations, in which higher sST2 concentrations were associated with adverse outcomes (19). Experimental data support an overall protective effect of IL33/ST2 signaling; activation of membrane-bound ST2L by IL33 leads to reduced hypertrophy and fibrosis (9). The genetic variants studied in our manuscript are located in membrane-bound ST2L, and we show that genetic variants in membrane-bound ST2L enhance both sST2 expression and also IL-33 responsiveness of ST2L itself. Thus, genetic variants likely influence both soluble and membrane-bound ST2. It may be that increased expression of ST2 reflects a compensatory response to cardiac stress, as seen with other molecules (such as BNP), in which the association of genetic variants with outcomes has the opposite directionality from circulating levels (39). We found strong evidence linking IL1RL1 genotypes with soluble ST2; on the other hand, the associations with clinical outcomes warrant further confirmation. Furthermore, because variants in this region are in high linkage disequilibrium, it is not possible to determine whether the effect is due solely to variants in IL1RL1 or neighboring loci within the linkage disequilibrium block, and identification of other causal variants requires additional study.

To our knowledge, our study is the first investigation to demonstrate associations between ST2 genotype and circulating human sST2 concentrations. We extended findings to in silico tissue expression: rs13001325 was associated with both sST2 concentrations and IL1RL1 gene expression in brain tissue. The directionality of the association was consistent among genotype, gene expression, and sST2 concentrations, lending additional weight to this finding. These data are consistent with prior gene expression studies, demonstrating significant sST2 mRNA levels in brain tissue compared with that in other organs (40).

We explored potential functional consequences of sST2 missense 
variants in our GWAS first by homology mapping the variants to the IL-1R. The A78E variant mapped to the surface of sST2 and extracellular ST2L domain, with the side chain oriented toward the hydrophobic protein interior and, based on homology with the IL-1 $\beta$ receptor, would be unlikely to disrupt the IL-33 binding site. Of the remaining 5 IL1RL1 coding mutations, 4 mapped to the TIR domain on membrane-bound ST2, an intracellular signaling domain common to all Toll and IL-1Rs. The A433T variant would be predicted to have the highest probability of directly influencing TIR domain-mediated signaling by ST2. This variant was located in "box 2," a region predicted by in silico docking studies to facilitate protein-protein interactions with the TIR domains of the adaptor proteins myeloid differentiation primary response gene 88 (MyD88) and MyD88 adaptor-like (Mal), leading to expression of proinflammatory cytokines (41).

In order to elucidate the functional consequences of these ILIRL1 nSNPs, we examined the effect of each of these missense mutations on sST2 expression in experimental studies. The extracellular A78E variant did not alter sST2 expression, suggesting that IL-33 binding to the extracellular ST2L domain was not altered by this variant. All other intracellular variants were associated with increased SST2 expression, indicating that signal transduction via the ST2L intracellular domain plays an important role in regulating sST2. This occurs via alterations in ST2 signaling pathways, including transcription factors NF- $\mathrm{BB}$ and AP-1 $(28,29)$, which are known to regulate sST2 expression, and the PI3K/AKT/mTOR pathway, which is known to regulate ST2L expression (30). The A433T and Q501R variants specifically demonstrated enhanced SST2 expression after IL-33 induction, which was blocked by both anti-ST2L mAb and anti-IL-1 $\beta$. This suggests that mTOR-mediated ST2L expression contributes to increased IL-33-induced SST2 expression observed in these variants. Interestingly, while Q501R and Q501K variants both result in positively charged amino acid substitutions, they have differential effects on signaling pathways. Q501R but not Q501K inhibited the PI3K/AKT/mTOR pathway and increased IL-33 responsiveness through increasing endogenous ST2L expression. Furthermore, we found that A433T and Q501R interact with the PI3K-p85 subunit and may regulate AKT signaling by modulating the interaction of the ST2L TIR domain with other receptor tyrosine kinase or PI3K subunits $(32,42)$.

Due to the high degree of linkage disequilibrium at the IL1RL1 locus, it is not possible to determine where the true signal originates using population-based data. However, these experimental findings suggest that missense variants underlie, at least in part, the genetic determinants of sST2 concentrations. We found other sST2 variants located in neighboring genes within the 400-kb linkage disequilibrium block but not within the IL1RL1 locus. These variants could theoretically contribute to ST2 regulation via trans effects and will need further study. Given our focus on common variants, we expect that other variants may influence ST2 concentrations, each with a small-to-modest effect size.

A recent study showed that deletion of the proximal ST2 promoter disrupts fibroblast-specific sST2 expression but does not affect serum sST2 levels (43). These results may be reconciled with our findings by considering that ST2 promoter usage appears to be highly cell-type dependent and that fibroblasts may not be the major source of circulating sST2. Human endothelial cells, immune cells, and lung epithelial cells are known to express high levels of sST2 and might be the major source of circulating sST2 $(40,44)$. We found that IL1RL1 intracellular variants associated with higher sST2 concentrations on a population level were also associated with higher sST2 protein levels in KU812 human basophils as well as in A549, U937, and Jurkat T cells. This suggests that the regulation of sST2 expression by IL1RL1 missense variants can occur in human immune and epithelial cells.

Several limitations deserve mention. The relative contributions of clinical and genetic factors to variation in sST2 are estimates, and clearly unmeasured factors not included in our analysis may also influence sST2 concentrations. Specifically with regards to heritability estimates, the influence of shared genes and shared environmental exposures is often difficult to distinguish. However, we found no significant correlation of SST2 concentrations among spousal pairs, which suggests that shared environmental factors are probably less important.

In summary, our findings demonstrate substantial heritability of sST2 concentrations and identify a robust association with genetic variants within or near the IL1RL1 gene. In molecular studies, IL1RL1 missense variants identified using a population-based approach induced sST2 expression via direct effects on ST2L signaling and modulation of ST2 promoter activity. Studies elucidating the functional consequences of genetic variation in SST2-related genes may lead to a better understanding of the ST2 pathway and its possible influences on immune and inflammatory diseases, including cardiovascular disease.

\section{Methods}

\section{Study sample}

The FHS Offspring study is a prospective, observational, community -based study (45). Children (and spouses of the children) of FHS original cohort participants were recruited in 1971 and have since been followed with serial examinations. Soluble ST2 concentrations were measured on stored frozen blood specimens collected at the sixth examination cycle (1995-1998). A total of 2,991 participants were included in this analysis.

\section{Laboratory testing}

Blood samples collected after an overnight fast were centrifuged for storage at $-70^{\circ} \mathrm{C}$. sST 2 concentrations were measured using a high-sensitivity sandwich immunoassay (Presage ST2; Critical Diagnostics) (46). The assay is stable over time and has a lower detection limit of $2 \mathrm{ng} / \mathrm{ml}$. Within-run and total coefficients of variation are $\leq 2.5 \%$ and $\leq 4.0 \%$, respectively (46).

\section{Genome-wide genotyping and imputation}

Genotyping was conducted using the Affymetrix 500K mapping array and the Affymetrix $50 \mathrm{~K}$ gene-focused MIP array (dbGaP study accession phs000342.v7.p7). Genotypes from the Affymetrix 500K mapping array were called using Chiamo (47). For imputation of genotypes to the HapMap set of 2.5 million SNPs (CEU population, release 22, build 36; http://hapmap.org), a hidden Markov model was used, as implemented in MACH (version 1.0.15) (48).

In silico association of genetic variants with gene expression, clinical outcomes, and other cardiovascular biomarkers

The top 10 sST2 SNPs with pairwise $r^{2}<0.2$ were searched against a collected database of expression SNPs (eSNPs) to examine relations with cis gene expression levels across different tissue types (Supplemental Methods). Because circulating ST2 concentrations are associated with mortality and cardiovascular events, we performed look-ups in large GWASs for association of sST2 genetic variants with these clinical outcomes in 
the CHARGE (22) and CARDIoGRAM consortia (details in the Supplemental Methods) (23).

Comparative modeling of ST2 and missense variants. Homology modeling of the ST2 protein structure was used to analyze missense variants using SWISS-MODEL (49). Crystal structures of either the T1R domain of IL-1R-associated protein (IL-1RAcP, protein data bank ID 1T3G) or IL-1R type II (IL-1RII) bound to IL-1 $\beta$ and IL-1RAcP (protein data bank ID 3O4O) were used as templates for models of ST2.

\section{Cell culture studies}

Cloning and plasmids. For details, please see the Supplemental Methods. In brief, the human basophil cell line KU812 was maintained in RPMI 1640 (Invitrogen) containing 10\% FBS. HEK293 cells were cultured in DMEM (Invitrogen) containing 10\% FBS. Full-length WT human ST2L was obtained via RT-PCR from KU812 cDNA. pCR-Blunt II Topo vector (Invitrogen) was used as intermediate vector. Mutations were introduced using PCR-based mutagenesis. Full-length ST2L cDNA was then subcloned into PCDH-CMV-MCS-EF1-GFP-T2A-Puro lentiviral vector (System Biosciences). All constructs were verified by DNA sequencing.

Lentivirus preparation and cell line creation. Lentiviral constructs were introduced with psPAX and PMD2.G packaging and envelope vectors into 293 TN cells. Culture media were harvested after 72 hours, and lentiviral particles were concentrated by ultracentrifugation (if destined for in vivo delivery) or Peg-IT reagent (if destined for cell line creation). Stable cell lines were created by transducing KU812 cells with concentrated lentivirus and subsequent puromycin selection by limited dilution in 96-well plates. IL1RL1 variant constructs were generated using PCR-based mutagenesis. The 5-mut construct has a minor variant on codon Q501 with CAG to AGG (Q501R) and encodes ST2L protein containing variants A433T, T549I, Q501R, and L551S. Expression was confirmed via Western blotting for ST2 and/or His-taq as appropriate.

ST2 promoter constructs and promoter assays. We used PCR with specific primers to amplify the different regions upstream of exon 1a (distal) and exon $1 \mathrm{~b}$ (proximal) of the human IL1RL1 gene from the DNA of the BAC clone (RP11-315O22). ST2 proximal and distal promoters fragments containing different lengths of sequences were inserted between the SacI and NheI sites of the pGL3-basic vector (Promega) containing the entire coding sequences of firefly luciferase with specific primers. For ST2 promoter assays, KU812 cells expressing ST2L variants were transfected by using Purefection (System Biosciences) with $500 \mathrm{ng}$ of plasmids. After 24-hour incubation, cells were harvested in lysis buffer and assayed for luciferase activity.

Real-time PCR. Total RNA was isolated using TRIzol reagent (Invitrogen). cDNA was synthesized from $1 \mu \mathrm{g}$ of total RNA and random hexamers using the TaqMan Reverse Transcription Kit (Applied Biosystems). Realtime PCR was performed in a 7300 Real-Time PCR system (Applied Biosystems). All reactions were performed in duplicate. The relative amount of $s S T 2$, ST2L, or IL33 mRNA was normalized to HPRT.

ELISA. For basal sST2 protein expression level analysis, KU812 cells expressing WT or IL1RL1 variants were cultured in serum-free RPMI 1640 medium for 24 hours. The media were collected for ELISA analysis for sST2 (Presage ST2, Critical Diagnostics), IL-1 $\beta$ (R\&D System), and IL-33 (R\&D System). For signal activation analysis, cells were subjected to serum starvation for 8 hours and then treated with PBS or IL-33 for 30 minutes. Cell lysates were for ELISA detection (Cell Signaling Technology) of phospho-NF-кB p65 (Ser536), phospho-c-Jun (Ser63), phospho-AKT (Thr308), and phospho-STAT3 (Tyr705).

\section{Statistics}

Soluble ST2 concentrations were log transformed due to right-skewed distribution. To examine the association of sST2 with clinical covariates, a forward selection regression model was used, with $P<0.05$ for entry (details in the Supplemental Methods). Analyses were performed using SAS, version 9.1.3 (SAS Institute).

Heritability. Variance-component models were used to estimate the heritability of sST2 using Sequential Oligogenic Linkage Analysis Routines (50). Heritability estimates were age and sex adjusted and also multivariable adjusted (age, sex, systolic blood pressure, antihypertensive medication use, diabetes mellitus, and smoking status). Clinical covariates suspected to be potential confounders were selected based on prior studies $(13,34,51)$. Secondary analyses are detailed in the Supplemental Methods.

GWAS. Associations of genetic variants and sST2 concentrations were tested using linear mixed-effects models to accommodate pedigree data under an additive genetic model, with covariates used for heritability analysis. Genome-wide association analyses were performed using R (52) and implemented using the lmekin function in the kinship package (53). Results were considered genome-wide significant at $P<5 \times 10^{-8}$. Soluble ST2 concentrations (back transformed from log-transformed data) were estimated by genotype for the top SNP.

\section{Study approval}

Participants provided informed consent, and the study was approved by the Institutional Review Board at Boston University Medical Center.

\section{Acknowledgments}

This work was partially supported by the National Heart, Lung and Blood Institute's Framingham Heart Study (N01HC-25195), its contract with Affymetrix Inc. for genotyping services (N02-HL-6-4278), and NIH grants R01-NS017950-28 and R01-HL093328-01 (to R.S. Vasan), R01 HL092930 (to R.T. Lee), R01-HL086875 (to T.J. Wang), and 1K23-HL116780 (to J.E. Ho). J.E. Ho is supported by a Boston University School of Medicine Department of Medicine Career Investment Award. Measurement of sST2 was performed by Critical Diagnostics Inc. S. Cheng is supported by an award from the Ellison Foundation. W.-Y. Chen is supported by awards from the Taiwan National Science Council (101-2917-I-564-057) and an AHA postdoctoral fellowship (13POST16940030). A. Dehghan is supported by a NWO grant (veni, 916.12.154) and the EUR fellowship. The analyses reflect intellectual input and resource development from the Framingham Heart Study investigators participating in the SNP Health Association Resource (SHARe) project. A portion of this research used the Linux Cluster for Genetic Analysis (LinGA-II) funded by the Robert Dawson Evans Endowment of the Department of Medicine at Boston University School of Medicine and Boston Medical Center. Please see Supplemental Acknowledgments for CHARGE and CARDIoGRAM consortium details.

Received for publication October 4, 2012, and accepted in revised form June 27, 2013.

Address correspondence to: Thomas J. Wang, Division of Cardiovascular Medicine, Vanderbilt University Medical Center, 2220 Pierce Ave., 383 Preston Research Building, Nashville, Tennessee 37232-6300, USA. Phone: 615.936.1717; Fax: 615.936.2029; E-mail: thomas.j.wang@vanderbilt.edu. Or to: Richard T. Lee, Brigham and Women's Hospital, Partners Research Facility, 65 Landsdowne Street, Cambridge, Massachusetts 02139, USA. Phone: 617.768.8282; Fax: 617.768.8270; E-mail: rlee@partners.org. 
1. Iwahana $\mathrm{H}$, et al. Different promoter usage and multiple transcription initiation sites of the interleukin-1 receptor-related human ST2 gene in UT-7 and TM12 cells. Eur J Biochem. 1999;264(2):397-406.

2. Miller AM. Role of IL-33 in inflammation and disease. J Inflamm (Lond). 2011;8(1):22.

3. Oboki $\mathrm{K}$, et al. IL-33 is a crucial amplifier of innate rather than acquired immunity. Proc Natl Acad SciUS A. 2010;107(43):18581-18586

4. Arend WP, Palmer G, Gabay C. IL-1, IL-18, and IL-33 families of cytokines. Immunol Rev. 2008; 223:20-38.

5. Kurowska-Stolarska M, et al. IL-33 induces antigen-specific IL-5 + T cells and promotes allergic-induced airway inflammation independent of IL-4.J Immunol. 2008;181(7):4780-4790.

6. Pastorelli L, et al. Epithelial-derived IL-33 and its receptor ST2 are dysregulated in ulcerative colitis and in experimental Th1/Th2 driven enteritis. Proc Natl Acad Sci US A. 2010;107(17):8017-8022.

7. Xu D, et al. IL-33 exacerbates antigen-induced arthritis by activating mast cells. Proc Natl Acad Sci U S A 2008;105(31):10913-10918.

8. Weinberg EO, et al. Expression and regulation of ST2, an interleukin-1 receptor family member, in cardiomyocytes and myocardial infarction. Circulation. 2002; 106(23):2961-2966

9. Sanada S, Hakuno D, Higgins LJ, Schreiter ER, McKenzie ANJ, Lee RT. IL-33 and ST2 comprise a critical biomechanically induced and cardioprotective signaling system. J Clin Invest. 2007; 117(6):1538-1549.

10. Seki K, et al. Interleukin-33 prevents apoptosis and improves survival after experimental myocardia infarction through ST2 signaling. Circ Heart Fail. 2009;2(6):684-691.

11. Weinberg EO, Shimpo M, Hurwitz S, Rouleau J-L, Lee RT. Identification of serum soluble ST2 receptor as a novel heart failure biomarker. Circulation. 2003 ; 107(5):721-726.

12. Boisot S, et al. Serial sampling of ST2 predicts 90-day mortality following destabilized heart failure. J Card Fail. 2008;14(9):732-738.

13. Ky B, et al. High-sensitivity ST2 for prediction of adverse outcomes in chronic heart failure. Circ Heart Fail. 2011;4(2):180-187.

14. Mueller T, Dieplinger B, Gegenhuber A, Poelz W, Pacher R, Haltmayer M. Increased plasma concentrations of soluble ST2 are predictive for 1-year mortality in patients with acute destabilized heart failure. Clin Chem. 2008;54(4):752-756

15. Manzano-Fernández S, Mueller T, Pascual-Figal D, Truong QA, Januzzi JL. Usefulness of soluble concentrations of interleukin family member ST2 as predictor of mortality in patients with acutely decompensated heart failure relative to left ventricular ejection fraction. Am J Cardiol. 2011; 107(2):259-267.

16. Shah RV, Chen-Tournoux AA, Picard MH, van Kimmenade RRJ, Januzzi JL. Serum levels of the interleukin-1 receptor family member ST2, cardiac structure and function, and long-term mortality in patients with acute dyspnea. Circ Heart Fail. 2009; 2(4):311-319.

17. Shimpo M, et al. Serum levels of the interleukin-1 receptor family member ST2 predict mortality and clinical outcome in acute myocardial infarction.
Circulation. 2004;109(18):2186-2190.

18. Sabatine MS, et al. Complementary roles for biomarkers of biomechanical strain ST2 and N-terminal prohormone B-type natriuretic peptide in patients with ST-elevation myocardial infarction. Circulation. 2008;117(15):1936-1944.

19. Wang TJ, et al. Prognostic utility of nove biomarkers of cardiovascular stress: the Framingham Heart Study. Circulation. 2012; 126(13):1596-1604

20. Akhabir L, Sandford A. Genetics of interleukin 1 receptor-like 1 in immune and inflammatory diseases. Curr Genomics. 2010;11(8):591-606.

21. Coglianese EE, et al. Distribution and clinical correlates of the interleukin receptor family member soluble ST2 in the Framingham Heart Study. Clin Chem. 2012;58(12):1673-1681

22 . Walter S, et al. A genome-wide association study of aging. Neurobiol Aging. 2011;32(11):2109.e15-e28.

23. Schunkert H, et al. Large-scale association analysis identifies 13 new susceptibility loci for coronary artery disease. Nat Genet. 2011;43(4):333-338.

24. Liefeld T, Reich M, Gould J, Zhang P, Tamayo P, Mesirov JP. GeneCruiser: a web service for the annotation of microarray data. Bioinformatics. 2005 21(18):3681-3682.

25. Moffatt MF, et al. A large-scale, consortium-based genomewide association study of asthma. NEngl J Med. 2010;363(13):1211-1221.

26. Slack JL, et al. Identification of two major sites in the type I interleukin-1 receptor cytoplasmic region responsible for coupling to pro-inflammatory signaling pathways. J Biol Chem. 2000 275(7):4670-4678

27. Weinberg EO, et al. Expression and regulation of ST2, an interleukin-1 receptor family member, in cardiomyocytes and myocardial infarction. Circulation. 2002;106(23):2961-2966

28. Tare $\mathrm{N}$, et al. KU812 cells provide a novel in vitro model of the human IL-33/ST2L axis: functional responses and identification of signaling pathways. Exp Cell Res. 2010;316(15):2527-2537.

29. Schmitz J, et al. IL-33, an interleukin-1-like cytokine that signals via the IL-1 receptor-related protein ST2 and induces T helper type 2-associated cytokines. Immunity. 2005;23(5):479-490.

30. Turnquist HR, et al. IL-1beta-driven ST2L expression promotes maturation resistance in rapamycin-conditioned dendritic cells. J Immunol. 2008 181(1):62-72

31. Choi YS, et al. Interleukin-33 induces angiogenesis and vascular permeability through ST2/TRAF6mediated endothelial nitric oxide production. Blood. 2009;114(14):3117-3126.

32. Drube S, et al. The receptor tyrosine kinase c-Kit controls IL-33 receptor signaling in mast cells. Blood. 2010;115(19):3899-3906.

33. Januzzi JL, et al. Measurement of the interleukin family member ST2 in patients with acute dyspnea: results from the PRIDE (Pro-Brain Natriuretic Peptide Investigation of Dyspnea in the Emergency Department) study. J Am Coll Cardiol. 2007; 50(7):607-613.

34. Pascual-Figal DA, et al. Soluble ST2, high-sensitivity troponin $\mathrm{T}$ - and $\mathrm{N}$-terminal pro-B-type natriuretic peptide: complementary role for risk stratification in acutely decompensated heart failure.
Eur J Heart Fail. 2011;13(7):718-725

35. Gudbjartsson DF, et al. Sequence variants affecting eosinophil numbers associate with asthma and myocardial infarction. Nat Genet. 2009; 41(3):342-347.

36. Hunt KA, et al. Newly identified genetic risk variants for celiac disease related to the immune response. Nat Genet. 2008;40(4):395-402.

37. Smyth DJ, et al. Shared and distinct genetic variants in type 1 diabetes and celiac disease. $N$ Engl J Med. 2008;359(26):2767-2777.

38. Benagiano $\mathrm{M}$, et al. T helper type 1 lymphocytes drive inflammation in human atherosclerotic lesions. Proc Natl Acad Sci USA. 2003; 100(11):6658-6663.

39. Newton-Cheh C, et al. Association of common variants in NPPA and NPPB with circulating natriuretic peptides and blood pressure. Nat Genet. 2009; 41(3):348-353.

40. Mildner M, et al. Primary sources and immunological prerequisites for sST2 secretion in humans. Cardiovasc Res. 2010;87(4):769-777.

41. Basith S, Manavalan B, Govindaraj RG, Choi S. In silico approach to inhibition of signaling pathways of Toll-like receptors 2 and 4 by ST2L. PLoS One. 2011;6(8):e23989.

42. Laird MH, et al. TLR4/MyD88/PI3K interactions regulate TLR4 signaling. J Lenkoc Biol. 2009; 85(6):966-977.

43. Lipsky BP, Toy DY, Swart DA, Smithgall MD, Smith DE. Deletion of the ST2 proximal promoter disrupts fibroblast-specific expression but does not reduce the amount of soluble ST2 in circulation. Eur I Immunol. 2012;42(7):1863-1869.

44. Zhao J, et al. Lysophosphatidic acid increases soluble ST2 expression in mouse lung and human bronchial epithelial cells. Cell Signal. 2012;24(1):77-85.

45. Kannel WB, Feinleib M, McNamara PM, Garrison RJ, Castelli WP. An investigation of coronary heart disease in families. The Framingham offspring study. Am J Epidemiol. 1979;110(3):281-290.

46. Dieplinger B, et al. Analytical and clinical evaluation of a novel high-sensitivity assay for measurement of soluble ST2 in human plasma - the Presage ST2 assay. Clin Chim Acta. 2009;409(1-2):33-40.

47. Wellcome Trust Case-Control Consortium. CHIAMO. Genetic Analysis Software Web site. https:// mathgen.stats.ox.ac.uk/genetics_software/ chiamo/chiamo.html. Accessed July 22, 2013.

48. Li Y, Willer C, Sanna S, and Abecasis GR. Genotype imputation. Annu Rev Genomics Hum Genet. 2009; 10:387-406

49. Schwede T, Kopp J, Guex N, Peitsch MC. SWISSMODEL: An automated protein homology-modeling server. Nucleic Acids Res. 2003;31(13):3381-3385.

50. Almasy L, Blangero J. Multipoint quantitative-trait linkage analysis in general pedigrees. Am J Hum Genet. 1998;62(5):1198-1211.

51. Rehman SU, Mueller T, Januzzi JL. Characteristics of the novel interleukin family biomarker ST2 in patients with acute heart failure. J Am Coll Cardiol. 2008;52(18):1458-1465

52. Chen MH, Yang Q. GWAF: an R package for genome-wide association analyses with family data. Bioinformatics. 2010;26(4):580-581.

53. http://cran.r-project.org/src/contrib/Archive/ kinship/. Accessed on August 20, 2013. 